\title{
Constructions participiales absolues et liaisons de prédications : au-delà du lien syndétique
}

\author{
Havu, Eva \& Pierrard, Michel \\ Université de Helsinki \& Vrije Universiteit Brussel \\ eva.havu@helsinki.fi \& michel.pierrard@vub.ac.be
}

\begin{abstract}
Résumé. Historiquement reliée à l'ablatif absolu de la grammaire latine, la proposition ou construction absolue (CPA) qui associe « deux termes dans une relation prédicative, sans expliciter par une marque formelle leur rapport entre elles, ni avec le reste de l'énoncé » (Riegel et al. 2009 : 356) a été évoquée par nombre de linguistes, mais c'est surtout Suzanne Hanon (1989) qui y a consacré un ouvrage entier. Le second terme peut prendre la forme d'un adjectif (Il se promenait, la tête basse), d'un groupe prépositionnel (Il se promenait les mains dans les poches) ou encore d'un participe prédicatif, le cas que nous examinerons ici (Son amie rentrant demain, il a nettoyé l'appartement).. L'examen des traits définitoires de la construction participiale absolue révèle des caractéristiques paradoxales : D'une part, elle se situe dans un rapport de dépendance/ subordination par rapport à une prédication régissante, et même si elle constitue une prédication (une « phrase »), il $\mathrm{y}$ a toujours absence de flexion verbale marquant l'accord de personne, le temps, le mode ou l'aspect. D'autre part, il y a absence de lien/ marque syndétique du rapport entre la CPA et la proposition régissante : Aucun connecteur de type adverbial, prépositionnel ou conjonctionnel n'indique donc obligatoirement la relation, ce qui engendre un fonctionnement apparemment autonome.Notre contribution se donne pour but de préciser le mode de fonctionnement et le statut des constructions participiales absolues.
\end{abstract}

\begin{abstract}
The absolute clause, or construction, historically related with the Latin "ablativus absolutus", forms a predicative relation between two terms without explicitly marking their mutual relation or their relation with the rest of the enunciation (cf. Riegel et al. $2009: 356$ ). In French, it has been examined by several linguists, especially Suzanne Hanon (1989) who has dedicated a whole monography to the subject. The second term of the construction can be an adjective (Il se promenait, la tête basse "He walked his head low"), a prepositional phrase (Il se promenait les mains dans les poches "He walked his hands in his pockets ») or a predicative participle (Son amie rentrant demain, il a nettoyé l'appartement "His friend coming tomorrow he cleaned the flat"). This last type will be examined in this paper. The definitory features of absolute participial constructions reveal paradoxical characteristics: on one hand they are in a dependency /subordinate relation with the governing predication and although they form a predication (a "clause") they always lack verbal flexion marking person, time, mood or aspect. On the other hand, there is no syndetic mark indicating the relation between absolute participial constructions and the main clause. Since no adverbial, prepositional or conjunctional connective is needed, they seem to function autonomously. The objective of our paper is to specify the ways of using and defining absolute participial constructions.
\end{abstract}


Historiquement reliée à l'ablatif absolu de la grammaire latine, la proposition ou construction absolue a été évoquée dans de nombreuses études (voir e.a. Arnavielle (2003, 2009), Blanche-Benveniste (1998, 2006), Combettes (1998), Haff (2010, 2012), Halmøy (2006, 2008), Mouret (2011), Müller-Lancé (1998)) et Suzanne Hanon (1989) y a même consacré un ouvrage entier. Elle est généralement définie de la façon suivante : "Les constructions absolues associent deux termes dans une relation prédicative, sans expliciter par une marque formelle leur rapport entre elles, ni avec le reste de l'énoncé » (Riegel et al., 2009 : 356). Le second terme peut prendre la forme d'un adjectif (il se promenait, la tête basse), d'un groupe prépositionnel (il se promenait les mains dans les poches) ou encore d'un participe prédicatif, le cas que nous examinerons ici :

(1a) $[\ldots]$ on but avec enthousiasme à la santé des phoques en général et bientôt, l'alcool exaltant les affects, voici qu'on l'invitait même à passer la nuit là s'il le souhaitait, [...] (Echenoz, p. 100)

(1b) Puis, le premier enthousiasme passé, Ferrer avait commencé de se méfier : [...] (Echenoz, p. 7475)

L'examen des traits définitoires de la construction participiale absolue (CPA) révèle des caractéristiques paradoxales :

- D'une part, la CPA se situe dans un rapport de dépendance/ subordination par rapport à une prédication régissante (les propositions absolues «présentent un caractère de dépendance, puisqu'elles ne peuvent exister seules » (Hanon, 1989 : 59)). De fait, bien qu'elle constitue une prédication (une «phrase »), il y a toujours absence de flexion verbale marquant l'accord de personne, le temps, le mode ou l'aspect.

- D'autre part, il y a absence de marque syndétique du rapport entre la CPA et la proposition régissante : le terme absolu a ici le sens « qui n'a pas de liaison explicite avec le support du complément » (Grevisse \& Goosse, 2008 : §253). Aucun connecteur de type adverbial, prépositionnel ou conjonctionnel n'indique donc obligatoirement la relation, ce qui engendre un fonctionnement apparemment autonome (Hanon (1989) parle de «nexus »).

Notre contribution se donne pour but de préciser le mode de fonctionnement et le statut des CPA.

\section{Absence de lien syndétique et (non) marquage du lien}

\subsection{Absence de lien syndétique et liaison de prédications}

Quel est le rapport entre une marque syndétique et une liaison de prédications ? Dans une approche typologique de la jonction de propositions, Lehmann (1988:210) identifie divers «struments connectifs » (il parle de « connective device »). Ainsi, sur l'axe syndétique de la parataxe (Ibid. : 210-211) il mentionne des pronoms déictiques ou anaphoriques, des verbes anaphoriques et des adverbes ou des séquences adverbiales, à côté des particules connectives. Cependant, le rapport paratactique peut aussi prendre une forme asyndétique (veni, vidi, vici). Pour ce qui concerne la subordination (Ibid. : 212-213), il répertorie comme «strument connectif » non seulement les mots subordonnants (tels les conjonctions), mais aussi des séquences anaphoriques (au moment où, de manière que). De plus, la subordination peut, elle aussi, adopter le mode asyndétique par l'emploi de formes non finies. D'autres auteurs, tel Dixon (2011), soulignent également des multiples possibilités de liaison sans marqueur : 


\begin{abstract}
two clauses are simply apposed, without a specific marker and are by inference taken to indicate one of the semantic types of clause linking [...]. Which kind of linkage is involved will be inferable from placement of stress; intonation pattern; tense, aspect, modality, mood and similar specifications in the clauses; and from the pragmatics of discourse. (Dixon, $2011: 37$ )
\end{abstract}

L'absence de marqueur syndétique n'exclut donc pas l'instauration d'un lien entre prédications.

\title{
1.2 (Sous-)spécification du lien
}

Si l'asyndèse n'élimine pas nécessairement le lien, elle aura cependant un impact sur la (sous-) spécification de celui-ci : l'asyndèse tend à rendre le lien entre les prédications plus opaque (Pierrard \& Havu, 2015), même si d'autres formes de liaison entre les prédications peuvent contrebalancer la sous-spécification et que le marquage de ce rapport peut se faire au moyen de divers indices autres que les «struments connectifs » classiques. Ces différents indices présentent-ils toutefois tous les mêmes propriétés connectives? La question se pose donc de la caractérisation des différents modes de liaison interpédicationnel, établis par ces marques diverses :

(2a) Contournez le mur, vous trouverez un sentier étroit.

(2b) Contournez le mur et vous trouverez un sentier étroit.

(2c) Quand vous contournez le mur, vous trouverez un sentier étroit.

(2d) Après avoir contourné le mur, vous trouverez un sentier étroit.

(2e) En contournant le mur, vous trouverez un sentier étroit.

Dans l'ensemble des exemples (2a-e), un lien de dépendance entre les deux prédications sera induit par l'interlocuteur. La différence d'appréhension des énoncés n'est toutefois pas seulement le produit de l'absence ou de la présence d'une marque formelle de connexion (absence dans $2 \mathrm{a}\rangle\langle$ présence dans $2 \mathrm{~b}-\mathrm{e}$ ) mais aussi et surtout de l'impact variable des divers types d'indices formels, syndétiques (2b-c) ou non (2de), sur l'appréhension du lien entre les prédications (2b : «coordonnant », $2 \mathrm{c}$ : « subordonnant », 2d-e : formes non fléchies du verbe). Il convient dès lors de distinguer les énoncés non seulement selon l'absence ou la présence d'un marquage explicite, syndétique ou non, de la liaison mais également selon les différents types d'indices de liaison et de prendre en considération les modalités ou les contraintes que ceux-ci imposent au lien interprédicationnel et aux prédications impliquées.

\section{Type de lien et interprétation des CPA}

Le rapport de dépendance entre la CPA et sa régissante n'est pas marqué de manière syndétique, mais cela ne signifie donc pas nécessairement qu'il y a absence d'indices de jonction et que ceux-ci n'imposent pas des contraintes interprétatives à l'ensemble. Déjà, l'absence de finitude de la forme verbale dans le cas des absolues est un indice impliquant une forme de dépendance envers un autre prédicat. Une configuration structurellement proche des absolues, les co-prédicatives participiales adjointes (cf. Havu \& Pierrard, 2008, 2014), révèle un autre type de lien entre prédication seconde et prédication régissante.

\subsection{Le rôle de l'ancrage nominal et de la position dans les co-prédicatives adjointes}

Les configurations syntaxo-sémantiques co-prédicatives sont proches de celles étudiées ici. En particulier, le co-prédicat prendra souvent, comme dans les CPA, la forme d'un adjectif (pâle et muet, il se tenait à la porte), d'un syntagme prépositionnel (Léon, de bonne humeur, accueillit Paul avec un large sourire) et, surtout, les structures participiales y sont aussi très fréquentes (Havu \& Pierrard, 2009). Dans ces coprédicatives, le mode de liaison entre les prédications est spécifié par le N support du co-prédicat (Christian 
Bîmes, dans (3a-b)), qui occupe une position argumentale (éventuellement intrasyntagmatique) dans la régissante :

(3a) S'étonnant de voir cinq licenciés faire appel à la justice pour demander des éclaircissements financiers, Christian Bîmes a décidé de contre-attaquer : « [...] » (Le Figaro, 11-12.12.2004/31)

(3b) Étonné de voir cinq licenciés faire appel à la justice pour demander des éclaircissements financiers, Christian Bîmes a décidé de contre-attaquer : «[...]».

Le $\mathrm{N}$ support mais aussi la position de la co-prédicative conditionneront l'interprétation du lien interprédicationnel. Celle-ci pourra être de type circonstanciel (3a-b : «Comme/ alors qu'il s'étonne/ est étonné ») ou descriptif (3c-d : «Christian Bîmes, qui s'étonne/ est étonné ») :

(3c) Christian Bîmes, s'étonnant de voir cinq licenciés faire appel à la justice pour demander des éclaircissements financiers, a décidé de contre-attaquer.

(3d) Christian Bîmes, étonné de voir cinq licenciés faire appel à la justice pour demander des éclaircissements financiers, a décidé de contre-attaquer.

La mise en place d'une liaison de prédications par le double rôle d'un $\mathrm{N}$ de la co-prédication n'est pas un cas unique dans la jonction de prédications. Ce mode de connexion se retrouve en effet dans d'autres types de constructions, par exemple chez les relatives $(4 a)^{1}$. Dans de nombreuses langues comme en français, la jonction de propositions au moyen du double rôle d'un $\mathrm{N}$ sera réalisée sous la forme d'un marqueur syndétique relatif (4a), qui permet de dissocier les fonctions de ce $\mathrm{N}$ dans les deux prédications. Dans certaines langues cependant (4b), il n'y a pas nécessairement dissociation des rôles par la présence du marqueur relatif :

(4a) Ma sœur sortait du cinéma/ J'ai rencontré ma sœur qui sortait du cinéma.

(4b) I have read the book you gave me yesterday.

La liaison de la prédication seconde avec la prédication régissante peut donc s'opérer à travers un ancrage nominal. C'est le cas des co-prédicats participiaux adjoints qui sont donc syntaxiquement liés à la régissante par le biais de l'intégration de leur thème à la structure argumentale de la régissante (comme le démontre sa cliticisation en 4c-c'). Or, c'est précisément ce qui les sépare, du moins en apparence ${ }^{2}$, des CPA, dont le thème est, par définition, non intégré dans la régissante (cf. le caractère « absolu » de la prédication seconde, qui n’a « pas de liaison explicite avec le support du complément »(Grevisse \& Goosse, 2008 : §253)). De surcroit, ce thème est non cliticisable (cf. 4d-d') :

(4c) J'aime le café chaud.

(4c') Je l'aime chaud, le café.

(4d) Le café étant enfin chaud, Pierre se mit à préparer ses toasts.

(4d') *Il étant enfin chaud, le café, Pierre se mit à préparer ses toasts.

L'opposition entre les co-prédicats participiaux adjoints et les CPA semble radicale et tranchée. L'est-elle autant qu'il n'y paraît à première vue ?

\subsection{Type de lien et type de CPA}

La saisie du type de rapport avec la régissante est d'autant plus importante que les chercheurs qui ont examiné le tour, tels Hanon (1989) et Mouret (2011), séparent essentiellement deux sortes de CPA selon qu'elles sont (a) issues d'une structure à prédication seconde (5a : type A (Hanon, 1989) $)^{3}$ ou (b) le produit d'une circonstancielle (5b : type B (Ibid. $)^{4}$ :

(5a) Marie est assise, les yeux fermés.

(5b) La porte fermée, Marie s'en alla. 
Dans (5a), la CPA sera plutôt rapprochée d'un type particulier de structure co-prédicative à ancrage nominal (elle a les yeux fermés; cf. déjà Hanon 1989), dans la mesure où son thème est lié par une relation de possession à un argument de la prédication régissante $(\text { les yeux }=\text { ses yeux }=\text { les yeux de Marie })^{5}$. Envers la prédication régissante, elle dénote alors une manière d'être (Mouret, 2011 : 51). Dans (5b), par contre, la participiale absolue est issue, selon Hanon (1989), d'une circonstancielle (comme/ parce que la porte était fermée, Marie s'en alla). Elle n'a plus d'ancrage nominal et dénote donc une circonstance (Mouret, 2011 : $51)$.

Les auteurs cités rattachent des fonctions spécifiques à cette répartition : les occurrences du type A « décrivent des états généralement associés à la description des êtres humains ou des objets (donc des états simultanés par rapport à l'action principale) », tandis que celles du type B mettent « en relation de cause à effet ou en relation de temporalité deux prédications » (Hanon, 1989: 63), soit des interprétations d'ordre descriptif ou circonstanciel, qui sont similaires de celles relevées dans le cas des co-prédicatives adjointes (cf. §2.1.). Pour Mouret (2011 : 51), le statut syntaxique des deux types serait même différent (une phrase pour le type B ou un GN prédicatif pour le type A) : « [...] les formes qui dénotent une circonstance dans laquelle s'inscrit la situation dénotée par la phrase dans laquelle elles s'insèrent, qui seront analysées comme des phrases à part entière, de celles liées à un argument dans la phrase par une relation de possession qui dénotent une manière, qui seront analysées comme des GN prédicatifs » (Mouret, 2011 : 51).

\subsection{Méthodologie et questions de recherche}

La répartition proposée par Hanon (1989) et Mouret (2011), ainsi que le rapprochement suggéré avec les co-prédications participiales dans le cas du type A demandent d'examiner de plus près les diverses sortes de rapports liant la CPA à la régissante et l'impact de celles-ci sur le statut sémantico-syntaxique et sur l'interprétation de la construction absolue.

Les objectifs de la présente contribution sont donc de deux ordres :

(a) décrire les différents types de liens rattachant la CPA à la régissante en examinant si la bipartition proposée peut être maintenue ${ }^{6}$;

(b) évaluer leur impact sur le statut sémantico-syntaxique/ l'interprétation des CPA ainsi que sur leur rapport avec les co-prédicatives participiales adjointes.

Notre étude sera basée sur l'exploitation d'un corpus de 178 occurrences de CPA ainsi que sur les exemples donnés par Mouret et Hanon. Pour répondre aux questions de recherche, nous prendrons en considération les paramètres du type de participe (présent (PPant) ou passé (PPé) $)^{7}$ et de la position (anté- ou postposition par rapport au prédicat régissant).

\section{Les CPA descriptives (type A)}

Selon Hanon (1989) et Mouret (2011), les traits caractérisant le type A sont les suivants :

(a) il est issu d'une structure à prédication seconde, où le thème de celle-ci est lié par une relation de possession à un argument de la prédication régissante ;

(b) la CPA y a un sens descriptif : elle décrit « des états généralement associés à la description des êtres humains ou des objets (donc des états simultanés par rapport à l'action principale) » (Hanon, 1989 : 63) ;

(c) son statut syntaxique est celui d'un GN prédicatif : les configurations, « liées à un argument dans la phrase par une relation de possession qui dénotent une manière, [...] seront analysés comme des GN prédicatifs » (Mouret, 2011 : 51).

Examinons la pertinence des différentes propriétés énoncées. 


\subsection{Relation de possession : acception restreinte}

Dans une acception restreinte du trait (a), les occurrences convoquées indiquent une relation de possession de type partie-tout « entre (au moins) un GN inclus dans la construction absolue d'une part, obligatoirement introduit par l'article défini, et un référent saillant du procès dont la construction dénote une manière d'autre part, le premier étant interprété comme une partie inaliénable (en général une partie du corps) du second interprété comme le possesseur de cette partie, avec une paraphrase toujours possible au moyen d'une structure à attribut de l'objet formée sur le verbe avoir » (Mouret, 2011: 54). Le GN de la prédication absolue, nécessairement introduit par l'article défini, sera interprété comme une partie inaliénable d'un référent de la régissante. La construction implique aussi un rapport d'attribution en avoir, au sein duquel la structure absolue adjointe constitue en fait un apposé/ prédicat composé qui se rapporte à un support qui remplit la fonction sujet dans préd1 avec une valeur partie/ tout (ou possession inhérente) (6a).

(6a) Il dort, la bouche ouverte.

= Il dort et [il (a) la bouche ouverte]

Les occurrences de type (6a) sont relativement rares dans le corpus examiné (4 occurrences, toutes avec un participe passé) : elles apparaissent en antéposition et surtout en postposition :

(7a) Les jambes tendues, vous contractez les abdominaux et soufflez. (La Deux - Fit Tonic 13/07/2012 $-7 \mathrm{~h} 30)$

(7b) $\quad[\ldots]$ : je passais des heures debout, le front collé au verre, à jouer à me jeter dans le vide. (Nothomb, p. 150)

(7c) J'ai passé une bonne partie de la nuit debout, le front appuyé contre la fenêtre à regarder le Parc des Expositions. (Gavalda, p. 182)

(7d) J'ai toujours adoré cette formule qui correspond si bien au jeu des acteurs dans les films de samouraïs, quand ils s'adressent à leur chef, la voix traumatisée par un respect surhumain. (Nothomb, p. 172)

Dans quelle mesure les occurrences relevées confirment-elles les propriétés (b) et (c) ?

\subsubsection{Trait (b) : la valeur descriptive}

Selon Mouret, les CPA de ce type «ne dénotent pas une circonstance à proprement parler, c'est-à-dire une situation, mais une manière d'un procès » (Mouret, $2011: 54$ ).

3.1.1.1. S'il est vrai qu'elles ne dénotent pas une circonstance (valeur temporelle, causale, hypothétique ou concessive), elles n'expriment pas non plus un sens adverbial de manière. Diverses observations amènent à les séparer des adverbiaux de manière :

(a) La réponse à la question «comment» ne permet pas d'identifier univoquement les adverbiaux de manière :

(8a) Il dort profondément/ Il dort comment ? (adverbial de manière)

(8b) Il boit son thé chaud/ Il le boit comment? (attribut de l'objet)

(8c) Il est en pleine forme/ Il est comment? (attribut du sujet)

(b) Les CPA peuvent fonctionner comme attribut (8d) ou (comme Mouret le signale d'ailleurs également) en coordination avec une épithète détachée (8e), contrairement à un adverbial de manière :

(8d) Il est/ a la bouche ouverte/ *Il est/ a profondément.

(8e) A... n'a pas bronché depuis sa découverte : très droite sur sa chaise, les deux mains reposant à plat sur la nappe de chaque côté de son assiette. (Mouret, 2011 : exemple 11a) 
Les CPA n'expriment donc pas la manière dont se développe un procès, mais bien la « manière d'être » d'un participant à l'action : «états généralement associés à la description des êtres humains ou des objets (donc des états simultanés par rapport à l'action principale)» (Hanon, 1989 : 63). Cela est souligné par le fait que, si la CPA peut fonctionner en antéposition, de par le lien qui l'unit à l'agent de l'action dont il exprime la manière d'être $(9 a)$, cela ne fonctionne pas avec l'adverbial de manière dans la même position (9b) :

(9a) La bouche ouverte, il dort profondément

(9b) *Profondément, il dort la bouche ouverte.

3.1.1.2. Cela signifie-t-il pour autant qu'une lecture circonstancielle est totalement exclue dans ce cas de figure ? L'interprétation circonstancielle de manière dans (6a) est bien pertinente, mais ce genre de lecture est influencé par divers paramètres :

(a) le type d'événements :

(6a) Il dort, la bouche ouverte.

(6a') Elle est assise les yeux fermés.

Dans (6a), la manière d'être du sujet peut aussi apparaître comme une modalité de l'événement, ce qui n'est pas le cas dans (6a'), dans la mesure où le sémantisme du prédicat régissant ne l'y invite pas.

(b) la position :

(10a) Les yeux embués par les larmes, elle ne voyait pas bien qui entrait dans la pièce.

(7a') Vous contractez les abdominaux et soufflez, les jambes tendues,

(10b) Il n'a pas bougé d'une ligne : [accroupi, la tête baissée, les avant-bras s'appuyant sur les cuisses, les deux mains pendant entre les genoux écartés]. (Robbe-Grillet : 183 ; apud Mouret, ex. 15a)

(10a') Elle ne voyait pas bien qui entrait dans la pièce, les yeux embués par les larmes.

La construction absolue antéposée dans (7a) pourrait aussi être paraphrasée par une circonstancielle de temps (Une fois que vous avez tendu les jambes...). Elle basculera nettement vers le circonstanciel si le verbe de la principale exprime une activité dont la manière d'être de l'actant, énoncée par la participiale absolue, peut constituer la cause (10a). En position finale par contre (7a'), ces constructions ont indubitablement une valeur d'addition descriptive, comme l'illustre clairement l'exemple (10b) provenant de Mouret, encore qu'une lecture circonstancielle ne soit pas, ici aussi, exclue (10a').

Par ailleurs, la valeur circonstancielle s'impose évidemment si les constructions absolues initiales ou finales sont accompagnées d'une expression de temps du type une fois, à peine, quel que soit le verbe de la principale et quelle que soit la position occupée : Une fois les jambes tendues / le front collé au verre, je passais des heures debout; Je passais des heures debout, une fois les jambes tendues / le front collé au verre. Aucun exemple de ce genre avec une CPA de type A n'apparaît cependant dans notre corpus.

\subsubsection{Trait (c) : statut de GN prédicatif}

Pour justifier son analyse du type A en tant que GN prédicatif, Mouret (2011) avance plusieurs arguments.

(a) Les emplois attributifs ou coordonnés à un constituant prédicatif sont naturels pour les CPA de type A. De fait, ils «peuvent être coordonnées avec ou sans conjonction à des catégories prédicatives sans sujet réalisé, par exemple à des formes au participe passé ou encore des GA » (Mouret, 2011 : 55)

(10b) Il n'a pas bougé d'une ligne : [accroupi, la tête baissée, les avant-bras s'appuyant sur les cuisses, les deux mains pendant entre les genoux écartés]. (Robbe-Grillet : 183 ; apud Mouret, ex. 15a)

(8e) A... n'a pas bronché depuis sa découverte : très droite sur sa chaise, les deux mains reposant à plat sur la nappe de chaque côté de son assiette. (Robbe-Grillet : 62 ; apud Mouret, ex. 11a) 
L'observation est correcte et souligne la convergence fonctionnelle de ces entités dans l'énoncé. Toutefois, cela ne semble pas être un argument suffisant pour justifier l'appréhension de ces tours comme des GN prédicatifs. De manière générale en effet, une même fonction peut être remplie par des entités de statut différent :

(11) Patrick est un homme bon, affable et qui ne ferait pas de mal à une mouche.

Dans (11), la relative est coordonnée à des adjectifs et ils remplissent tous une fonction spécifiante auprès du même N. Cependant, leur statut syntaxique est différent (adjectif ou proposition).

(b) Les CPA de type A peuvent être employées «non seulement comme ajouts à la phrase, ou à d'autres catégories telles que le GN (12a), mais aussi comme attributs du sujet (12b-c), avec une reprise acceptable au moyen de la proforme clitique prédicative le ». (Mouret, $2011: 56$ )

(12a) Mais pour apercevoir le salon depuis la table - ou par une fenêtre, le côté des hangars - il faudrait occuper [la place de Franck : le dos tourné au buffet]. (Robbe-Grillet : 69 ; apud Mouret, ex. 16a)

(12b) Alors elle relève le visage, ferme le livre - qu'elle pose à portée de main sur la table basse - et reste le regard fixé droit devant elle, vers la balustrade à jours et les bananiers de l'autre versant, bientôt invisible dans l'obscurité. (Robbe-Grillet : 16-17 ; apud Mouret, ex. 16c)

(12c) Est-elle restée/demeurée le regard fixé droit devant elle?/ Elle l'est restée/demeurée effectivement, et longtemps. (apud Mouret, ex. 16d)

Ici aussi, l'observation est correcte et souligne une fois de plus la convergence fonctionnelle entre ces entités dans un énoncé donné. Mais l'argument ne démontre pas le statut de GN prédicatif des CPA en question dans la mesure où des entités de statut clairement propositionnel peuvent aussi remplir la fonction :

(12d) Il reste toujours qui il était dans notre jeunesse/ Il le reste toujours, malgré tous les événements qui ont marqué notre vie.

(12e) Il m'a dit ceci : que tu partiras bientôt.

Dans (12d), il s'agit d'une proposition subordonnée qui remplit la fonction d'attribut. Dans (12e), c'est aussi une proposition subordonnée conjonctive qui, dans ce cas-ci, remplit la fonction d'ajout.

(c) Le flottement du quantifieur tous, caractéristique de la fonction sujet, n'est pas autorisé selon Mouret (2011) dans ce cas (13b) :

(13a) Penchée sur le côté, toutes ses pinces à la main, elle refait sa coiffure avant de venir à table. (ex. 17a ; Mouret, $2011: 56$ )

(13b) *Penchée sur le côté, ses pinces toutes à la main, elle refait sa coiffure avant de venir à table. (ex. $17 \mathrm{~b} ;$ ibid.)

L'impossibilité du flottement du quantifieur tous ne semble toutefois pas absolue :

(13c) Elles relèvent le visage, ferment le livre et restent tous les regards fixés sur la portel les regards tous fixés sur la porte.

\subsection{Relation de possession : acception large}

Dans une acception large du trait (a), le rapport de possession qui lie le thème de la structure absolue à un argument de la régissante n'est plus nécessairement inaliénable. Selon Haff, dans une acception large, «the absolute construction depends on a relation of possession which can be stated in terms of "pertinence" between its subject and/or predicate and the matrix subject, viz. the possessor or owner of the inalienable element. This relation of pertinence $[\ldots]$ is usually expressed by a possessive or reflexive anaphor [...] » (2010 : 211 ; cf. Haff 2012 : 261). La CPA se trouvera aussi bien en position antéposée (6c) que postposée (6b) : 
(6b) Il est entré un cadeau caché dans chacune de ses poches.

[*Il a le cadeau caché dans chacune de ses poches]

(6c) Son amie rentrant demain, il a nettoyé l'appartement.

[*Il a l'amie rentrant demain]

La possession inaliénable n'est plus envisageable ici et le tour co-prédicatif en avoir n'est plus permis (*Il a le cadeau caché). A ce propos, il faut constater que l'exemple (13a), avancé par Mouret (exemple (17a) dans Mouret, 2011), est en réalité un exemple d'emploi au sens large de possession. Les tours exprimant une acception non intrinsèque de la valeur possessive sont aussi rares dans le corpus et on les trouve uniquement en position antéposée :

(14a) Mon vélo étant immobilisé depuis maintenant + oh mon dieu cinq jours (rire) que j' suis obligée d' prendre le métro + je suis catastrophée par le fait que les gens me bousculent(CFPP2000)

(14b) parce que mon mari ayant été au Viêtnam et tout euh je sais faire les nems. (ESLO2)

Dans ces cas, l'interprétation circonstancielle devient clairement prédominante, malgré le rapport de possession (14a : comme mon vélo est immobilisé, [...]). Le rapport cause/ conséquence est d'ailleurs explicité dans (14b). Cette valeur se maintiendrait également si la CPA occupait une position finale (Je suis obligée de prendre le métro, mon vélo étant immobilisé depuis maintenant cinq jours).

D'autre part, le statut de GN prédicatif de l'absolue est moins que jamais soutenable dans le cas de l'expression de la possession non intrinsèque. En particulier le flottement du quantifieur tous, caractéristique selon Mouret (2011) de la fonction sujet, est parfaitement autorisé ici (14c-d) :

(14c) Tous nos vélos étant immobilisés, nous sommes obligées de prendre le métro/ Nos vélos étant tous immobilisés, nous sommes obligées de prendre le métro.

(14d) Tous nos maris ayant été au Viêtnam, nous savons faire les nems/ Nos maris ayant tous été au Viêtnam, nous savons faire les nems.

En conclusion, la particularité du type A réside moins dans son supposé statut particulier de GN prédicatif que dans sa capacité dans certains cas à imposer une interprétation descriptive. Ce fonctionnement spécifique du type A semble par ailleurs lié au rapport particulier qu'entretient le GN thème de la construction absolue avec un GN argument de la prédication régissante.

\section{Les CPA circonstancielles (type B)}

Le type B sera également caractérisé selon Hanon (1989) et Mouret (2011) par différents traits spécifiques :

(a) les CPA du type B sont issues de circonstancielles (La porte fermée, Marie s'en alla => comme/ parce que la porte était fermée, Marie s'en alla);

(b) Elles dénotent une circonstance et mettent « en relation de cause à effet ou en relation de temporalité deux prédications » (Hanon, $1989: 63)$;

(c) Elles n'ont plus d'ancrage nominal et leur statut syntaxique est celui d'une phrase : « [...] les formes qui dénotent une circonstance [...] seront analysées comme des phrases à part entière » (Mouret, 2011 : 51).

\subsection{Configuration standard}

La configuration standard du type $\mathrm{B}$, qui correspond bien à une sorte de « circonstancielle réduite » (trait a), est caractérisée par une construction participiale absolue et une régissante qui n'ont aucun élément en 
commun. Le thème/ support de la co-prédication jouit d'une autonomie formelle et n'est lié, ni sémantiquement, ni syntaxiquement à un argument du prédicat régissant.

(15a) Les conséquences politiques d'un tel scrutin restant difficiles à évaluer, la plupart des partis ont laissé à leurs électeurs toute liberté de conscience. (Le Monde, 12-13.6.2005/6)

(15b) Certains d'entre eux pourraient même dater du III ${ }^{\mathrm{e}}$ siècle avant J.-C., ce qui constitue une énigme, l'enfouissement ne datant, lui, que du $1^{\text {er }}$ siècle. (Le Figaro, 7.12.2004/23)

(15c) La première surprise passée, il devint orange. (Nothomb, p. 141)

(15d) Lionel Jospin s'est présenté devant les députés, le traité pour le passage à l'Euro déjà signé.

Si aucun lien syntaxo-sémantique explicite ne lie les deux prédications, le fonctionnement autonome de la CPA peut néanmoins induire des relations d'ordre discursif entre le thème de la CPA et un argument de la prédication régissante, sous des constellations de divers ordres : lien de famille (chat/ souris), relation de cause/ effet (question/ réponse) :

(16a) Le chat parti, les souris dansent.

(16b) Je vais vous poser les dernières questions, chaque bonne réponse valant un point. (Müller-Lancé, 1998 : 257)

\subsubsection{Trait (b) : la valeur circonstancielle}

Dans ses emplois standard, le type B oriente vers une lecture circonstancielle. Les CPA visent alors à mettre « en relation de cause à effet ou en relation de temporalité deux prédications » (Hanon, 1989 : 63). Selon Mouret (2011), les participes présents « dénotent une situation entretenant avec la situation décrite par la phrase matrice une relation de cause à effet » (2011 : 52) et lorsqu'un adverbe approprié tel 'pourtant' la contredit, une concession. Quant aux constructions avec un participe passé, elles « dénotent toutes une situation qui précède temporellement celle décrite par la phrase dans laquelle elles s'insèrent » (Ibid.). Il s'agit d'un emploi fréquent dans le corpus. Nous examinerons d'abord les CPA avec un participe présent pour observer ensuite celles comprenant un participe passé.

$\left(1^{\circ}\right)$ Les constructions avec participe présent :

En antéposition, il s'agit effectivement quasi uniquement d'interprétations causales (17a-b), parfois explicitées au moyen d'un marqueur syndétique (17c); parmi les exemples antéposés, il est néanmoins possible de retrouver aussi de rares emplois appelant une interprétation temporelle (17d) ;

(17a) Les fonctionnaires étant priés de boire du thé plus ordinaire et de refuser les cadeaux, les commandes ont baissé. (Le Monde diplomatique, novembre 2015/4)

(17b) donc c'était un peu le dernier d'une 1- + d'une lignée de chiens de plus de quarante ans quoi enfin de voilà + mais c'est c'est + ça a été quand même un peu un vide mais + bon $+j$ - non c'est c'est oh j' peux pas dire que ça me manque + finalement c'est pas vraiment à Paris enfin + ça me manque d'une certaine façon parce que le mien étant de grande taille + euh c'était quand même + moi presque une heure et demie d' sortie tous les jours hein (CFPP2000)

(17c) [...] comme moi-même étant ouvrier puis ayant pas fait de grandes études [...] bon fallait que je me défende en tant qu'ouvrier [...] (ESLO2)

(17d) Les bases américaines étant parties, le personnel est resté pour l'essentiel. (ESLO1)

En postposition par contre, l'emploi circonstanciel argumentatif (18a-b) est plus fréquemment en concurrence avec des interprétations comme ajouts descriptifs (18c-d). Dans certains cas, la CPA participe à l'expression d'une successivité discursive, d'un enchaînement d'événements (18e-f) :

(18a) Ce jour-là, quelques gaillards s'activent à la construction d'une propriété, le gouvernement ayant promis des subventions. (Le Monde diplomatique, novembre 2015/4) 
(18b) Certains d'entre eux pourraient même dater du IIIe siècle avant J.-C., ce qui constitue une énigme, l'enfouissement ne datant, lui, que du $1^{\text {er }}$ siècle. (Le Figaro, 7.12.2004/23)

(18c) [...], la dernière rencontre de Paris, début octobre, a permis d'envisager une trêve durable dans le Donbass, avec le retrait effectif des armes lourdes, les deux parties acceptant par ailleurs de reporter les élections locales afin de mettre au point une solution institutionnelle. (Le Monde diplomatique, novembre 2015/6)

(18d) Elle [évolution] fait apparaître une intégration sans précédent des satellites aux autres sources de reconnaissance, de communication et de commandement - l'architecture d'ensemble dépendant de façon critique de télécommunications sûres et à haute capacité via satellites. (Le Monde, 14.6.2005/1)

(18e) [...] : un village vidé de ses habitants, puis rénové ou même partiellement reconstruit à l'ancienne, certains étant alors invités à revenir pour animer ce "village touristique » à la vie artificielle. (Le Monde diplomatique, novembre 2015/5)

(18f) [...] ou même ils travaillent ensemble encore les les les juifs étant plutôt les patrons bien sûr odans un premier temps en tous cas des Chinois puis après les Chinois devenant eux-mêmes + leurs leurs propres patrons hein mais y a eu cette connexion alors les Juifs d'Irak + c'est au début qu' j'habitais là je dirais donc $+\mathrm{y}$ a vingt-c- vingt vingt-cinq ans (CFPP2000).

$\left(2^{\circ}\right)$ Les constructions avec participe passé :

Elles sont moins fréquentes dans le corpus. L'antéposition indique souvent l'antériorité (avec des CPA tendant à la formule figée (19c)), mais elle peut aussi déboucher sur le rapport cause/ conséquence (19d) :

(19a) Qu'est-ce qui s'est passé en 1997 ? Dissolution de l'Assemblée Nationale. Un traité pour le passage à l'Euro déjà signé, Lionel Jospin voulait mettre des conditions strictes, notamment d'emploi. (Interview de François Hollande par Olivier Mazerolle, BFM TV 19/02/2012)

(19b) Pour retrouver le code d'accès à l'immeuble, ses mains se frayèrent un chemin sous ses vêtements : la gauche vers l'agenda glissé dans une poche intérieure, la droite vers ses lunettes enfouies dans une poche pectorale. Puis, le portail franchi, négligeant l'ascenseur, il attaqua fermement un escalier de service. (Echenoz, p. 9)

(19c) Comme Ferrer allait bientôt prendre l'habitude d'aller lui emprunter livres et films, il mettrait peu de jours à comprendre que Brigitte, le soir venu, rejoignait un radiotélégraphiste à menton carré, nez fusiforme et moustache en guidon. (Echenoz, p. 19)

(19d) Donc, il faut vraiment qu'ils soient distincts dans leur forme. De toute façon c'est deux époques radicalement différentes. Le film complètement adapté à ce parti pris, Jean-François a fait deux films dont la narration diffère réellement. (Première Fr, Mesrine : Interview de Vincent Cassel, 06/05/10)

Souvent, la valeur temporelle est soulignée par un marqueur (à peine dans 19e, une fois dans 19f) :

(19e) Le dispositif à peine installé, deux clients toxicomanes sont repérés, à proximité de ce que les enquêteurs appellent « la plage » (RTBF, Devoir d'enquête, « Charleroi au bord de l'overdose », 28/07/11)

(19f) Une fois la plaie suturée, le jeune homme pourra quitter les urgences. (TF1 - Sept à huit, $12 / 08 / 2012-19 h)$

La postposition est rare et n'apparaît pas dans notre corpus, mais ne semble pas impossible. Il s'agit aussi d'une CPA à valeur temporelle :

(19f') Le jeune homme pourra quitter les urgences, une fois la plaie suturée.

(19g) Il se rapellera à leur bon souvenir, le moment venu. 


\subsubsection{Trait (c) : statut phrastique}

La possibilité de «flottement » du quantifieur 'tous' dans le cas des thèmes des participes présents comme des participes passés confirme que ceux-ci peuvent être considérés comme des sujets de phrase (Mouret, $2011: 53-54)$.

(17c') Tous les fonctionnaires étant priés/ Les fonctionnaires étant tous priés de boire du thé plus ordinaire et de refuser les cadeaux, les commandes ont baissé.

(18a') Ce jour-là, quelques gaillards s'activent à la construction d'une propriété, tous les gouvernements ayant promis/ les gouvernements ayant tous promis des subventions.

Mais est-ce que cela marche toujours ? Dans le cas des participes passés, cela semble marcher moins bien dans certains cas :

(19c') Tous les films complètement adaptés à ce parti pris, Jean-François a fait des films dont la narration diffère réellement/ ?Les films tous adaptés à ce parti pris, Jean-François a fait des films dont la narration diffère réellement.

(19e') Tous les dispositifs installés/?Les dispositifs tous installés, deux clients toxicomanes sont repérés, à proximité de ce que les enquêteurs appellent « la plage » (RTBF, Devoir d'enquête, " Charleroi au bord de l'overdose », 28/07/11)

(19f') Toutes les plaies suturées/ ?Les plaies toutes suturées, le jeune homme pourra quitter les urgences. (TF1 - Sept à huit, 12/08/2012 - 19h)

Dans le cas du type B aussi, les interprétations sont plus variées que ne le laisse supposer la bipartition proposée par Hanon ou Mouret et le genre d'événements combinés ou de la position dans l'énoncé jouent un rôle capital. Quant à l'ancrage nominal, il semble en effet bien rompu dans le type $\mathrm{B}$, ce qui constituerait alors le trait distinguant nettement ce type du précédent. Cependant, ici également, des configurations spécifiques sont à relever, où la chose est beaucoup moins nette qu'il n’y paraît.

\subsection{Configurations spécifiques}

Au sein du type $B$ des configurations spécifiques sont relevées où un certain ancrage nominal est réinstauré, malgré le trait (a) qui implique l'absence de rapport syntaxo-sémantique entre les deux prédications. Cet ancrage prend la forme d'un rapport co-référentiel et parfois même anaphorique entre le thème de la CPA et un argument de la régissante.

$\left(1^{\circ}\right)$ Reprise coréférentielle d'un argument de la régissante dans la CPA. Ce cas de figure, très fréquent dans le corpus, apparaît essentiellement en postposition (20a-d), mais pas exclusivement (20e) :

(20a) [...] Et ce qui compte, d'ailleurs, est que dans l'un et l'autre cas, on constate la nécessité d'enseigner la démocratie à des gens pour qui ce n'est ni évident ni naturel - la démocratie étant, par essence, contraire à toute supposée « loi naturelle ». (Le Soir, 19.3 04)

(20b) La logique est évidente. Cette exonération de charges compense les frais, pour l'entreprise, de l'accueil en formation du jeune, cet accueil en formation s'arrêtant au diplôme. (FR3, Questions au gouvernement, 09.03.05)

(20c) Le gouvernement fédéral avait du pain sur la planche vendredi. Indépendamment du volet « soins de santé » il devait arbitrer le conflit entre socialistes et libéraux, les uns et les autres défendant deux dossiers fiscaux et financiers bien différents. (RTBF, JT de 19h30, 05.03.05)

(20d) Néanmoins, il est u peu tôt pour en évaluer l'impact définitif, les tabacologues estimant que les fumeurs abstinents depuis quelques mois seulement peuvent encore replonger. L'ex-consommateur n'étant considéré comme sevré qu'un an après avoir écrasé sa dernière cigarette. (Le Figaro, 10.12.03/12) 
(20e) Nous étant une grosse société à l'échelon national si vous voulez on a un appui technique [...] (ESLO1)

Si l'antéposition garde une interprétation causale (20e), la configuration en postposition développe typiquement, quoique pas exclusivement (20d), un apport descriptif à la régissante. Ceci est souligné par la fréquence d'une reprise spécifiée de l'argument de la régissante, sous une forme distributive (21a-b) ou focalisée (21c-f), fondant l'ouverture de la prédication descriptive :

(21a) Les enfants montèrent dans le train, chacun d'eux portant une petite valise brune.

(21b) On avançait plutôt vite, chacun sèchement secoué sur son traîneau par les accidents de terrain. (Echenoz, p. 53-54)

(21c) Je reviens toujours à mon cas, moi,j'ai des enfants petits [...] l'ainée ayant cinq ans [...]. (ESLO1)

(21d) on opposait bourgeois au campagnard, le bourgeois étant celui qui habite le bourg [...]. (ESLO1)

(21e) Depuis la mort d'al-Mouqrin, trois étrangers ont été assassinés en Arabie saoudite, un Britannique, un Irlandais et un Français, seuls les deux premiers meurtres ayant des motifs politiques. (Le Figaro, 7.12.2004/2)

(21f) Cette $46^{\mathrm{e}}$ édition sera marquée par la rivalité entre Airbus et Boeing, le constructeur américain voulant reconquérir la première place mondiale que lui a ravie en 2003 son concurrent européen. (Le Monde, 14.6.2005/20)

$\left(2^{\circ}\right)$ Instauration d'un rapport (co)référentiel entre le thème de la CPA et un argument de la régissante au moyen d'une reprise du premier par une proforme dans la prédication régissante. Ce genre de configuration est également fréquent et est produit, contrairement à la précédente, essentiellement en antéposition (21af), même si la postposition n'est pas exclue ( $21 \mathrm{~g}-\mathrm{j})$. Notons que dans ce dernier cas, la source se situe dans la régissante et la proforme anaphorique fait partie de la CPA :

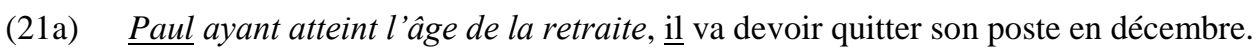

(21b) Son café étant enfin chaud, Pierre l'avala d'un trait.

(21c) Quant aux moustiques, leurs larves étant arrivées à maturité dans les flaques innombrables, $\underline{\text { ils }}$ attaquent de plus belle. (Echenoz, p. 64)

(21d) parce que ma femme étant fille de cheminot $[. .$.$] elle avait des réductions (ESLO2)$

(21e) A ce moment, les militants socialistes venaient de voter à 59\% en faveur de la Constitution européenne et $\underline{M}$. Fabius, partisan du non, ayant perdu, son congé aurait peu choqué. Mais M. Hollande avait à l'époque préféré jouer la carte du rassemblement. Avec le succès que l'on sait. (Le Monde, 1213.6.2005/12)

(21f) et on leur impose la langue française comme langue de travail, très bien, mais eux étant Brésiliens multilingues euh parfois il est plus aisé pour eux de d'exprimer des choses en anglais (CFPP2000

(21g) C'est lui [intermédiaire], selon nos informations, qui renoue, fin mars, le contact avec Philippe Brett, celui-ci affirmant alors à des journalistes qu'il informe le Quai d'Orsay et 'Libération' de ses discussions. (Le Monde, 14.6.2005/4)

(21h) « Le groupe Julia ne cessera plus, par la suite, d'annoncer de temps à autre (le 8 avril, le 13 avril...) une libération imminente, pour ensuite accuser le gouvernement d'avoir raté une occasion en distillant auprès de confrères, voire des amis de Florence, leur scénario des événements, dans lequel ils ont le beau rôle, leurs " informations » mêlant demi-vérités, mensonges et affirmations invérifiables, et se mêlant à des fuites gouvernementales. ». (Le Monde, 14.6.2005/4)

(21i) Il y a une autorité morale chez cette femme qui peut éventuellement s'étendre au Chili de Michelle Bachelet, elle aussi éduquée en Allemagne de l'Est. (Le Monde, 22-23.1.2006/16) 
(21j) près de quarante mille petites entreprises qui travaillent pour elle, ne faisant elle pratiquement que l'assemblage de toutes les pièces qui ont été fabriquées dans de petites entreprises de de trois quatre ou cinq ouvriers. (ESLO1)

\section{Conclusions}

L'examen à partir du corpus des constructions participiales absolues et de la bipartition suggérée par Hanon (1989) et Mouret (2011) nous a amené à avancer les observations suivantes :

(a) la bipartition stricte entre deux types de CPA doit être nuancée : les lectures circonstancielles et descriptives peuvent se retrouver dans les deux types distingués selon l'impact de divers paramètres discursifs, tels le genre d'événements combinés ou la position dans l'énoncé ;

(b) dans l'interprétation de la CPA, le lien entre le GN de la de prédication absolue et un argument de la régissante (sous diverses formes : relation partie/ tout ; rapport co-référentiel et parfois même anaphorique) s'est imposé comme un paramètre déterminant pour délimiter la signification du lien entre les prédications ;

(c) l'importance de l'ancrage nominal dans diverses sous-constructions absolues, que ce soit sous la forme de la possession inaliénable ou de la reprise anaphorique, étabit un lien étroit entre le fonctionnement des CPA et celui des co-prédications participiales adjointes.

A partir de ces constats, une étude plus systématique des paramètres évoqués devrait permettre d'affiner le mode de fonctionnement des CPA et de préciser leurs rapports avec les co-prédicatives participiales adjointes.

\section{Références bibliographiques}

Arnavielle, T. (2003). Le participe, les formes en -ant ; positions et propositions. Langages 37, 37-54.

Arnavielle, T. (2009). Le participe présent et le détachement : latitudes, difficultés, particularités. In Apothéloz, D., Combettes, B. \& Neveu, F. (éds), Les linguistiques du détachement, Berne : Peter Lang, 49-58.

Havu, E. \& Pierrard, M. (2008). Prédication seconde et lien appositif : intégration de prédicats et point d'ancrage. Faits de langues 31-32, 357-368.

Havu, E. \& Pierrard, M. (2009). Détachement et type d'opération linguistique : les participes présents détachés en position initiale et finale. In Apothéloz, D., Combettes, B. \& Neveu, F. (eds), Les linguistiques du détachement, Berne : Peter Lang, 305-316.

Havu, E. \& Pierrard, M. (2014). Les co-prédicats adjectivants. Berlin-Bern-Bruxelles : Peter Lang.

Blanche-Benveniste, C. (1998). Une fois dans la grammaire. Travaux de linguistique 36, 85-101

Blanche-Benveniste, C. (2006). Le rôle des participes passés dans la prédication. In Engwall, G. (éd.), Construction, acquisition et communication. Études linguistiques de discours contemporains, Stockholm: Université de Stockholm, 89-106.

Combettes, B. (1998). Les constructions détachées en français. Paris : Ophrys.

Furukawa, N. (2005). Pour une sémantique des constructions grammaticales. Thème et thématicité. Bruxelles: Duculot.

Dixon R. M. W. (2011). The Semantics of Clause Linking in Typological Perspective. In Aikhenvald, A. \& Dixon, R. M. W. (éds), The Semantics of Clause Linking: a cross-linguistic typology, Oxford (United Kingdom): Oxford University Press, 1-55.

Grevisse, M. \& Goosse, A. (2008) Le Bon Usage: Grammaire Française. Bruxelles : De Boek-Duculot.

Haff, M. H. (2012). On absolutes in French, German, and Norwegian. In Fabricius-Hansen, C. \& Truslew Haug, D. T. (éds.), Big events, small clauses : the grammar of elaboration, Walter de Gruyter, 259-286. 
Haff, M. H. (2010). A contrastive analysis of absolute constructions in French, German and Norwegian. Lingvistica investigationes, 208-224.

Halmøy, O. (2003) Le gérondif en français. Paris : Ophrys.

Halmøy, O. (2009) Les formes verbales en -ANT et la prédication seconde. Travaux de linguistique 57, 43-62.

Hanon, S. (1989). Les constructions absolues en français contemporain. Louvain-Paris : Peeters.

Lehmann, C. (1989). Towards a typologie of clause linkage. In Haiman, J. \& Thompson, S. A. (éds), Clause combining in grammar and discourse. Amsterdam \& New York : John Benjamins, 181-225.

Mouret, F. (2011). Deux types de constructions absolues dans La Jalousie de Robbe-Grillet. L'information grammaticale, 51-56.

Muller, C. (2011). Les interprétations sémantiques de la prédication seconde intégrée : les relatives prédicatives. Langue française 171, 101-116.

Müller-Lancé, J. (1998). Die Verwendung absoluter Konstruktionen in französischen Printmedien. In Hellrich, U. \& Klöden, H. (éds), Mediensprache in der Romania, Wilhelmsfeld: Gottfried Egert Verlag, 257-277.

Pierrard, M. \& Havu, E. (2015). Prédication seconde et (non-)marquage de la liaison. Langages 200, 15-30.

Riegel, M., Pellat, J.-C. \& Rioul, R. (2009 [1994]). Grammaire méthodique du français. Paris : Presses universitaires de France.

Corpus

a. Romans

Castillon, C- (2006). Insecte. Paris : Fayard.

Echenoz, J. (1999). Je m'en vais. Paris : Les Éditions de Minuit.

Gavalda, A. (1999). Je voudrais que quelqu'un m'attende quelque part. Paris : Le Dilettante.

Nothomb, A. (1999). Stupeur et tremblements. Paris : Albin Michel.

b. Journaux

2000-2004 : Exemples sporadiques rassemblés surtout dans L'Express, Le Monde, Libération, L'Express, Le Nouvel Observateur, Le Soir et Le Vif :

L'Express 8.6.2006 ;

Le Figaro 11-12.12 2004, 7.12.2004 ;

Le Monde 12-13.6.2005, 14.6.2005, 22-23.1.2006, 11-12.6.2006 ;

Le Monde Diplomatique, novembre 2015

Le Soir 19.32004 ;

Le Vif 13, 2004.

c. Corpus oral

2000-2010 : Exemples sporadiques recueillis à la télévision (surtout TV5) ;

2012 : Corpus 'étudiants' Bruxelles-Helsinki ; données recueillies au printemps et en été 2012 (journaux télévisés, débats, interviews, jeux (A2, Arte, Canal +, Club RTL, Direct8fr, HO, France 2, La Deux, La Une, La Trois, RTBF, RTL TV1, TF1)

CFPP : Discours sur la ville. Corpus de Français Parlé Parisien des années 2000 (CFPP2000).http://cfpp2000.univparis3.fr/

ESLO2 : Enquêtes SocioLinguistiques à Orléans (2014): http://eslo.huma-

num.fr/index.php/pagecorpus/pageaccescorpus 
ESLO1 : Enquêtes SocioLinguistiques à Orléans (1969 - 1974) : http://eslo.humanum.fr/index.php/pagecorpus/pageaccescorpus

${ }^{1}$ Voir à ce propos la distinction entre les relatives déterminatives, explicatives et prédicatives (cf. e.a. Furukawa, 2005, Muller, 2011).

${ }^{2}$ Haff (2010) a cependant examiné les possibilités de connexion entre la construction absolue et la phrase matrice : «I want to examine by what means (morphosyntactic, semantic or pragmatic) the absolute construction is connected to the subject of the matrix clause » $(2010: 211)$.

${ }^{3}$ Les types C (Pierre est torse nu) et $\mathrm{D}$ (Un jeune homme torse nu se promène dans le jardin) de Hanon (1989) peuvent en réalité être considérés comme des emplois particuliers du type A.

${ }^{4}$ Combettes (1998 : 20 ff.) fait également la différence entre «le schéma qui combine deux expressions dans une relation prédicative » et « ce que la tradition analyse comme une subordonnée participiale » où il n'y a pas « de structure à attribut de l'objet sous-jacente » (La porte fermée, nous sommes plus tranquilles) (Ibid. : 22).

${ }^{5}$ Ce type de rapport indirect peut aussi apparaitre dans le cas des participiales adjointes entre le thème de la coprédication et la fonction intrasyntagmatique exercée par celui-ci dans la régissante :

(a) Jeune, mon idole était Jouvet. (Le Figaro, 15.05.00/36).

(b) [Moi jeune] [mon idole était Jouvet]

${ }^{6}$ Hanon parle plus loin dans son ouvrage (1989 : 242) de valeurs intermédiaires entre les constructions du type A et B, c'est-à dire de cas où une construction du type A a une valeur circonstancielle (le contraire n'existerait pas, v. p. ex. p. 246)

${ }^{7}$ Cette contribution est centrée sur l'analyse du lien rattachant la CPA à une proposition régissante et à la bipartition des CPA suggérée par Hanon (1989) et par Mouret (2011). Elle n'approfondit pas le rôle des différents types de prédicats participiaux dans l'orientation de la diathèse ou dans l'expression de valeurs aspectuelles et modales. 